\title{
Quenching nematicon fluctuations via photo-stabilization
}

\author{
Nazanin Karimi ${ }^{1}$, Alessandro Alberucci ${ }^{1}$, Matti Virkki ${ }^{1}$, Arri Priimagi ${ }^{2}$, Martti Kauranen ${ }^{1}$ and Gaetano Assanto ${ }^{1,3}$ \\ ${ }^{1}$ Optics Lab, Department of Physics, Tampere University of Technology, 33101 Tampere - Finland \\ ${ }^{2}$ Department of Chemistry and Bioengineering, Tampere University of Technology, 33101 Tampere - Finland \\ ${ }^{3}$ NooEL-Nonlinear Optics and OptoElectronics Lab, University Roma Tre, 00146 Rome - Italy
}

Received March 07, 2016; accepted March 15, 2016; published March 31, 2016

\begin{abstract}
Light localization into optical spatial solitons can be achieved by launching optical beams in nonlocal nonlinear nematic liquid crystals. Such solitons often undergo undesired fluctuations of their trajectories. We demonstrate that partial polymerization in monoacrylate-doped nematic liquid crystals is effective in quenching such fluctuations in transverse space.
\end{abstract}

Spatial nonlocality in the optical response of dielectrics entails a number of benefits when dealing with nonlinear optics for signal processing and switching. It was recognized in the early days of nonlinear integrated optics that a diffusive hence nonlocal response, such as in thermo-optic materials, could lead to longitudinal optical feedback even in the absence of resonances, with consequent hysteresis versus input beam excitation [1-4] More recently, a nonlocal nonlinear response was found fit to stabilize beam self-confinement into twodimensional (2D) spatial solitons which, in local Kerr media with an intensity-dependent refractive index, are otherwise unstable and prone to filamentation and catastrophic collapse [5-10]. In the highly nonlocal limit in space, spatial optical solitons tend to mutually attract and exhibit long-range interactions, [11-12] as well as reduced modulational instability [13-14], incoherent character [15-16], and breathing oscillations [17-18]. The synergy of local and nonlocal effects in time was indicated as a route towards spatiotemporal light bullets [19].

Nematic liquid crystals (NLC) are molecular liquids with a substantial degree of orientational order, so much so that they exhibit a macroscopically (positive) uniaxial response, a reorientational nonlinearity, a large electrooptic effect as well as high nonlocality [20-21]. When an intense light beam with electric field $\mathbf{E}$ wave propagates through NLC, the induced microscopic dipoles tend to rotate under the action of torque:

$$
\Gamma=\varepsilon_{0}\left(n_{e}^{2}-n_{o}^{2}\right)(\mathbf{n} \cdot \mathbf{E})(\mathbf{n} \times \mathbf{E})
$$

with $\varepsilon_{0}$ the dielectric susceptibility of vacuum, $n_{e}$ and $n_{o}$ the refractive index eigenvalues for electric fields parallel and orthogonal to the optic axis $\mathbf{n}$, respectively. The resulting increase of the orientation angle $\theta$ between the

http://www.photonics.pl/PLP wavevector $\boldsymbol{k} \| z$ and $\mathbf{n}$ yields self-focusing through an increase in the extraordinary $(e)$-refractive index ruled by

$$
n_{e}(\theta)=\frac{n_{o} n_{e}}{\sqrt{\left(n_{o}^{2}-n_{e}^{2}\right) \sin ^{2} \theta+n_{e}^{2}}} .
$$

When self-focusing compensates for diffraction, a stable spatial soliton (a nematicon) is formed, with an associated graded-index waveguide wider that the beam generating it $[5,22]$. All-optical reorientation enables nematicon generation even at sub-mW power levels [2324], but such self-trapped beams are usually subject to dynamic instabilities due to the interplay between optical torque, thermal agitation, fluid convection [25], anisotropy [26], and anchoring conditions at the boundaries.

Transverse light localization through all-optical reorientation, in fact, is understood to locally increase the order parameter of this soft matter; this local field (alloptical) effect combines/competes with the bulk reorientation of the optic axis (molecular director) due to elastic interactions of the molecules (i.e., nonlocality) and their anchoring at the boundaries of the cell containing the liquid dielectric [21, 27]. As a narrow self-guided solitary beam is established in the nonlocal medium, the boundary conditions tend to restore the uniform distribution of a molecular director in the bulk, trying to relax lightinduced orientation distortion and thus pulling/pushing sideways the nematicon from its straight trajectory. The latter results in transverse fluctuations of the nonlinear beam versus time, particularly for propagation distances large enough to witness an appreciable reduction of its power through scattering losses.

Since nematicons are associated with light-induced waveguides potentially useful for guided-wave signal processing [28-29], a few strategies have been undertaken to reduce such transverse fluctuations in planar cells, e.g., by introducing external low-frequency electric fields along the direction of propagation [30-31], by varying the sample temperature [31], or by applying external magnetic fields [32]. Here, we report our first results on reducing/quenching nematicon transverse fluctuations through photo-induced stabilization in suitably doped 
materials, i.e., slightly altering the material itself as in Refs. [33-35].

We employed planar cells comprising two parallel glass slides separated by $100 \mu \mathrm{m}$ and with the inner interfaces coated with rubbed polyimide layers to anchor the molecular director at $\pi / 4$ with respect to $z$ in the plane $y z$. The liquid crystals we used were the standard nematic mixture 5CB doped with $10 \mathrm{~mol} \%$ monoacrylate (6-(4cyano-biphenyl-4'-yloxy) hexyl acrylate). The addition of monoacrylate into $5 \mathrm{CB}$ has been previously shown to increase reorientational nonlinearity compared to pure 5CB [34-35]. The unexposed NLC mixture exhibits the same optical properties as pure $5 \mathrm{CB}$, with $n_{o}=1.54$ and $n_{e}=1.70$.

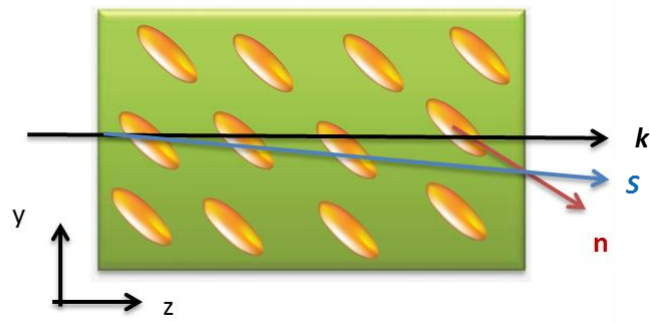

Fig. 1. Planar NLC cell for the study of nematicons. A $y$-polarized input beam can reorient nematic liquid crystals and produce self-focusing into a spatial soliton $S$ propagating in the plane $y z$ at a walk-off angle $\delta$ with respect to the wave vector $\boldsymbol{k} \| \boldsymbol{z}$. The director $\mathbf{n}$ was set at an initial orientation $\theta=\pi / 4$.

Nematicons were generated by launching a $3 \mathrm{~mW} y$ polarized continuous-wave Gaussian laser beam of wavelength $\lambda=1.064 \mu \mathrm{m}$ into a $3 \mathrm{~mm}$ long cell, monitoring the beam evolution in $y z$ with an optical microscope and a charge-coupled device camera able to image the out-ofplane scattered light.

Figure 2(a) shows a typical spatial soliton with a wavevector along $z$ but Poynting vector $S$ at the walk-off angle $\delta$, due to the birefringence of the positive uniaxial [36]. Figure 2(b) graphs various realizations of nematicon trajectories for a fixed excitation within a time interval $\Delta \mathrm{t}$ of about $4.5 \mathrm{~s}$ : due to transverse fluctuations, the solitary paths are distributed within a cone (seemingly distinct origins are just an artefact of the image fits).

Then, we illuminated the NLC sample with an ultraviolet (incoherent) continuous-wave light source (LED lamp) at $\lambda=365 \mathrm{~nm}$, with the power density $5 \mathrm{~mW} / \mathrm{cm}^{2}$, and monitored the soliton trajectories in fixed intervals of $4.5 \mathrm{~s}$ versus the UV exposure time. Noteworthy, since the dopant is a monomer and no photo-initiator was introduced in the mixture, no polymerization was expected as such. However, as shown in Fig. 3(a-b), the fluctuations were progressively quenched upon prolonged UV exposures. We attribute this behavior to weak partial polymerization of the acrylate groups. (a)

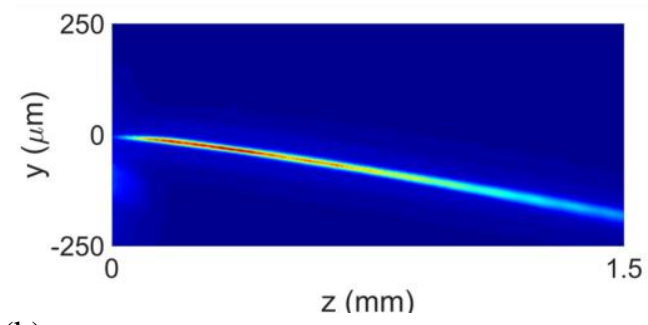

(b)

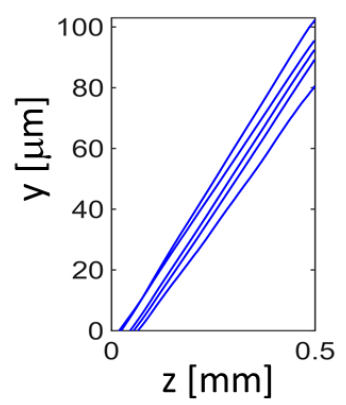

Fig. 2. (a) Color-coded photograph of a typical self-trapped spatial soliton excited by a $3 \mathrm{~mW}$ near-infrared beam. (b) Soliton trajectories of several nematicons randomly acquired within a time interval of about $4.5 \mathrm{~s}$.

From the graphs, a substantial decrease in the angular span of $(3 \mathrm{~mW})$ nematicon fluctuations with increasing UV exposure is apparent, with a path-cone of aperture nearly halved after 2 hours, as compared to the case before the exposure (Fig. 2(b)). Despite the extended UV illumination and reduced fluctuations, transverse light confinement was not appreciably affected, i.e. the nonlinear (reorientational) response of the sample did not undergo any degradation.
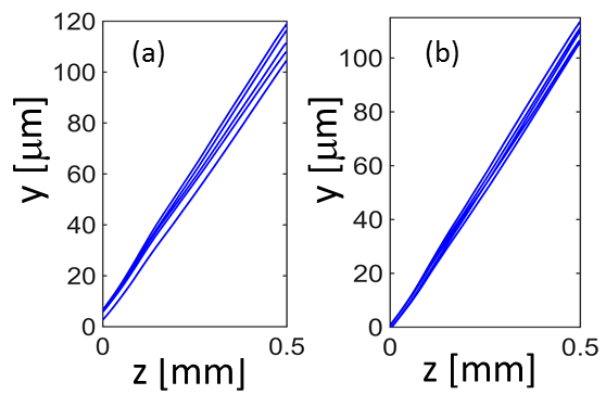

Fig. 3. Various realizations of nematicon trajectories in the plane $y z$ within a time interval $\Delta \mathrm{t}=4.5 \mathrm{~s}$, after $\mathrm{UV}$ exposure times of (a) $1.5 \mathrm{~h}$ and (b) $2 \mathrm{~h}$, respectively.

In conclusion, the tested new doped-NLC system exhibits lower transverse instabilities without penalty on all-optical reorientation. Such an improved response, consistent with the previous reports in polymer stabilized liquid crystals [33-35], can be attributed to modified 
viscosity or elasticity through partial polymerization, even though neither cross-linkers nor photo-initiators were present. These results are promising towards the alloptical generation of robust and temporally stationary guided-wave structures for optical signal processing.

N.K., A.A. and G.A. acknowledge the support from the Academy of Finland through the Finland Distinguished Professor grant no. 282858. A.P. and G.A. acknowledge the COST Action IC1208 of the European Union.

\section{References}

[1] G. Assanto, B. Svensson, D. Kuchibhatla, U.J. Gibson, C.T. Seaton, G.I. Stegeman, Opt. Lett. 11, 644 (1986)

[2] G.I. Stegeman, G. Assanto, R. Zanoni, C.T. Seaton, E. Garmire, A.A. Maradudin, R. Reinisch, G. Vitrant, Appl. Phys. Lett. 52(11), 869 (1988).

[3] G. Vitrant, R. Reinisch, J.Cl. Paumier, G. Assanto, G.I. Stegeman, Opt Lett. 14(16), 898 (1989).

[4] G. Assanto, G.I. Stegeman, Appl. Phys. Lett. 56(23), 2285 (1990)

[5] G.I. Stegeman, M. Segev, Science 286(5444), 1518 (1999).

[6] G. Assanto, M. Peccianti, IEEE J. Quantum Electron. 39(1), 13 (2003).

[7] D. Suter, T. Blasberg, Phys. Rev. A 48, 4583 (1993).

[8] W. Królikowski, O. Bang, Phys. Rev. E 63, 016610 (2000).

[9] C. Conti, M. Peccianti, G. Assanto, Phys. Rev. Lett. 91(7), 073901 (2003).

[10] J.H. Marburger, E. Dawes, Phys. Rev. Lett. 21, 556 (1968).

[11] M. Peccianti, K.A. Brzdąkiewicz, G. Assanto, Opt. Lett. 27(16), 1460 (2002).

[12] C. Rotschild, B. Alfassi, O. Cohen, M. Segev, Nature Phys. 2, 769 (2006).

[13] W. Krolikowski, O. Bang, J.J. Rasmussen, J. Wyller, Phys. Rev. E 64, 016612 (2001).

[14] M. Peccianti, C. Conti, G. Assanto, Phys. Rev. E 68, R025602 (2003).

[15] O. Cohen, H. Buljan, T. Schwartz, J.W. Fleischer, M. Segev, Phys. Rev. E 73, 015601R (2006).

[16] M. Peccianti, G. Assanto, Opt. Lett. 26(22), 1791 (2001).

[17] A.W. Snyder, D.J. Mitchell, Science 276, 1538 (1997).

[18] C. Conti, M. Peccianti, G. Assanto, Phys. Rev. Lett. 92(11), 113902 (2004).

[19] I.B. Burgess, M. Peccianti, G. Assanto, R. Morandotti, Phys. Rev. Lett 102, 203903 (2009).

[20] P.G. de Gennes, J. Prost, The Physics of Liquid Crystals (Oxford Science Publications, Clarendon Press, 2nd edition, 1993).

[21] I.C. Khoo, Liquid Crystals: Physical Properties and Nonlinear Optical Phenomena (Wiley, New York, 1995).

[22] M. Peccianti, G. Assanto, Phys. Rep. 516, 147 (2012)

[23] A. Piccardi, A. Alberucci, G. Assanto, Electron. Lett. 46(11), 790 (2010).

[24] A. Piccardi, A. Alberucci, G. Assanto, Phys. Rev. Lett. 104, 213904 (2010).

[25] J. Beeckman, K. Neyts, X. Hutsebaut, C. Cambournac, M. Haelterman IEEE J. Quantum Electron. 41(5), 735 (2005)

[26] E. Braun, L.P. Faucheux, A. Libchaber, Phys. Rev. A 48, 611 (1993).

[27] T. Wolinski, Private communication.

[28] M. Peccianti, G. Assanto, Opt. Lett. 26(21), 1690 (2001).

[29] A. Pasquazi, A. Alberucci, M. Peccianti, G. Assanto, Appl. Phys. Lett. 87, 261104 (2005).

[30] J.F. Henninot, J.F. Blach, M. Warenghem, J. Opt. A: Pure Appl. Opt. 10, 085104 (2008).

[31] A. Piccardi, Private communication.
[32] U. Laudyn, M. Karpierz, Private communication.

[33] J.F. Blach, J.F. Henninot, M. Petit, A. Daoudi, M. Warenghem, J. Opt. Soc. Am. B 5, 1122 (2007).

[34] Y. Aihara, M. Kinoshita, J. Wang, J.I. Mamiya, A. Priimagi, A. Shishido, Adv. Opt. Mat. 1(11), 787 (2013); http://doi.org/ 10.1002/adom.201300326

[35] J. Wang, Y. Aihara, M. Kinoshita, J.I. Mamiya, A. Priimagi, $\begin{array}{lllll}\text { A. Shishido, Sci. Rep. 5, } 9890 \quad \text { (2015); http://doi.org/ } & \end{array}$ 10.1038/srep09890

[36] M. Peccianti, A. Fratalocchi, G. Assanto, Opt. Express 12(26), 6524 (2004). 